\title{
Heterosis for Yield and Yield Attributing Traits in Barley (Hordeum vulgare L.)
}

\author{
Ankush Katiyar ${ }^{1}$, Aarti Sharma ${ }^{2 *}$, Akanksha Srivastava ${ }^{1}$, \\ Snehanshu Singh ${ }^{3}$ and S. R. Vishwakarma ${ }^{1}$
}

${ }^{1}$ Department of Genetics and Plant Breeding, Narendra Deva University of Agriculture and Technology, Kumarganj, Faizabad, UP, India

${ }^{2}$ Institute of Agricultural Sciences, Banaras Hindu University, Varanasi, UP, India

${ }^{3}$ Indian Institute of Wheat and Barley Research, ICAR, Karnal, Haryana, India

*Corresponding author

\section{A B S T R A C T}

\begin{tabular}{l} 
Ke y w o r d s \\
Barley, Standard \\
heterosis, \\
Heterobeltiosis \\
Article Info \\
$\begin{array}{l}\text { Accepted: } \\
\text { 04 September } 2020 \\
\text { Available Online: } \\
10 \text { October } 2020\end{array}$ \\
\hline
\end{tabular}

\section{Introduction}

Barley (Hordeum vulgare L.) is a cereal crop and belongs to Poaceae family. Barley is the fourth largest cereal crop produced around the world. It is a staple food in North Africa and parts of Asia where people consume barley in form of unleavened bread and porridges. Barley is used both as human food (bread, soups, stews, health drinks), animal feed and as source of malt for alcoholic beverages. In other uses, soft straw of barley plant is used in making livestock bedding. The versatile nature of barley makes it an important crop and thus demands increase in production of barley.

Study of heterosis in $\mathrm{F}_{1} \mathrm{~s}$ obtained from the hybridization program can be used to develop high yielding barley varieties. Heterosis refers to superiority of $\mathrm{F}_{1} \mathrm{~s}$ over their parents for different traits. The exploitation of heterosis has been extensively utilized in improving yield in crops. For a successful hybrid breeding programme, it is essential that a significant heterosis must be available in the 
$F_{1}$ populations and that a method is available for commercial seed production economically.

Understanding effectiveness of heterosis in plant breeding program; a study was undertaken to estimate heterosis in barley $\mathrm{F}_{1} \mathrm{~s}$ obtained by carrying out a crossing program in 10 barley genotypes following Line $\mathrm{x}$ Tester mating design.

\section{Materials and Methods}

Ten barley genotypes: NDB-3, Azad, RD2552, EC-667526, EC-667509, EC-667498, EC-667458, EC-667377, EC-667365, and EC-667454 were used as parental material in the present study.

The selected parental materials were subjected to hybridization program following Line $\mathrm{x}$ Tester mating design at Genetics and Plant Breeding Research farm of Narendra Deva University of Agriculture and Technology, Narendra Nagar, Kumarganj, Faizabad (U.P.) during Rabi 2013 and 2014. Seven lines (EC-667526, EC-667509, EC667498, EC-667458, EC-667377, EC-667365, EC-667454) were crossed with three testers (NDB-3, Azad, RD-2552) to generate $F_{1}$ seeds of each of the 21 crosses in Rabi 2013. The $F_{1}$ seeds were harvested, sundried and kept in cool and dry place. In Rabi 2014, the $21 \mathrm{~F}_{1} \mathrm{~s}$ along with 10 parents were sown in Randomized Block Design in two replications following a spacing of $25 \times 10 \mathrm{~cm}$. Package of practice was followed strictly to ensure a healthy crop stand.

Data was collected on randomly selected five plants from each of the 31 lines of two replications on traits viz., days to $50 \%$ ear emergence, days to $75 \%$ maturity, numbers of effective tillers per plant, number of grains per main ear, ear length, biological yield/plant, harvest index, 1000-grain weight and yield per plant. Analysis of variance was calculated following Panse and Sukhatme (1967). Heterobeltiosis was estimated following Fonseca and Patterson (1968). The formula used to estimate heterobeltiosis and standard heterosis is given below:

Heterobeltiosis $(\mathrm{HB} \%)=\frac{\overline{\mathbf{F}}_{\mathbf{1}}-\overline{\mathbf{B P}}}{\overline{\mathbf{B P}}} \times \mathbf{1} \mathbf{O O}$ Standard heterosis $(\mathrm{SH} \%)=\frac{\overline{\mathbf{F}_{1}}-\overline{\mathbf{S V}}}{\overline{\mathbf{S V}}} \times \mathbf{1 0 0}$

C.D. = S.E. $x \mathrm{t}($ at error d.f. $) ;$ S.E. $=(2 \mathrm{Me} / \mathrm{r})^{1 / 2}$

Where, C.D $=$ critical difference; S.E $=$ standard error; $\mathrm{Me}=$ mean sum of square of error; $r=$ number of replications; d.f.= degree of freedom; $\overline{\mathrm{SV}}=$ Mean value of the standard variety; $\overline{\mathrm{BP}}=$ Mean value of the better parent.

\section{Results and Discussion}

Analysis of variance for all traits except ear length was observed to be significant in the present study (Table-1). Early maturity is desirable hence negative heterosis is required for days to $50 \%$ ear emergence and days to 75\% maturity. Hybrids EC-667454×Azad and EC-667526×Azad, displayed negative heterosis over check variety and EC667498×RD-2552, EC-667509×Azad showed negative heterobeltiosis for days to $50 \%$ ear emergence. Out of 21; three cross combinations viz., EC-667526×NDB-3, EC$667377 \times$ NDB-3, EC-667377×RD-2552 found to be significant and negative for better parent and only one cross combination viz., EC$667377 \times$ NDB-3, showed negative significant standard heterosis for days to $75 \%$ maturity (Table-2). Lal et al., (2018) observed negative heterobeltiosis for early maturity in their work. An increase in number of productive tillers/plant can positively affect grain yield per plant hence positive heterosis is required 
for this trait. However, in the present finding only four of the cross combinations showed positive significant heterobeltiosis and standard heterosis viz., EC-667377 $\times$ RD2552, EC-667377 x NDB-3, EC-667377 $x$ Azad and EC-667498 $\times$ NDB-3. These findings are in conformity with the work of Lal et al., (2018).

For ear length, none of the cross combinations showed positive standard heterosis. Three cross combinations showed positive significant heterobeltiosis viz., EC-667365 $\times$ Azad, EC-667498 × RD-2552 and EC-667498 $\times$ NDB-3. For standard heterosis, no cross combination showed significant heterotic effect in desirable direction.

For number of grains per spike, positive significant heterosis is required. Out of 21 crosses, three cross combinations showed desirable positive significant heterobeltiosis viz., EC-667365×RD-2552, EC-667526×RD2552 and EC-667509 $\times$ RD-2552. Similarly for standard heterosis, two cross combinations showed positive significant heterosis: EC$667526 \times \mathrm{RD}-2552$, and EC-667509 $\times \mathrm{RD}-$ 2552. Amer et al., (2012), Mahmoud (2006), Eid (2006) and Mansour (2016) observed similar findings in their work. Significant and positive heterosis is desirable for 1000-grain weight. Cross combinations EC-667365×RD2552 and EC-667526×NDB-3 showed positive heterobeltiosis and cross combinations EC-667509 × Azad, EC-667526 $\times$ NDB-3, and EC-667365×RD-2552 showed significant positive standard heterosis. ElBawab (2003), Eid (2006) and Mansour (2007) have also reported heterosis in positive and negative direction for 1000-grain weight.

For biological yield per plant, significant positive heterobeltiosis were found in six crosses: EC-667377 × RD-2552, EC-667365 $\times$ RD-2552, $667526 \times$ RD-2552, EC-667498 $\times$ RD-2552, EC-667454 × RD-2552, EC$667454 \times$ Azad. And EC-667526 × RD-2552, EC-667458 $\times$ NDB-3, EC-667509 $\times$ RD2552, EC-667509 $\times$ NDB-3 showed positive significant heterosis over check variety. For harvest index, none of the crosses showed significant positive heterosis over better parent. While significant positive heterosis, was recorded in only one cross EC667526×NDB-3. Parashar et al., (2019) observed similar findings for biological yield and harvest index in their work.

Table.1 Analysis of variance of 21 hybrids for yield and yield attributing traits of barley

\begin{tabular}{|l|c|c|c|}
\hline Traits & Replications & Treatments & Error \\
\hline df & 1 & 30 & 30 \\
\hline Days to 50\% flowering & 5.82 & $14.48^{* *}$ & 2.62 \\
\hline Days to maturity (75\%) & 1.30 & $15.53^{* *}$ & 2.33 \\
\hline Number of productive tillers/plant & 0.03 & $0.52^{* *}$ & 0.12 \\
\hline Ear length (cm) & 0.02 & 0.91 & 0.39 \\
\hline Grains / ear & 0.15 & $46.44^{* *}$ & 2.97 \\
\hline 1000- grain weight (g) & 0.001 & $14.50^{* *}$ & 1.40 \\
\hline Biological yield / plant (g) & 0.80 & $8.90^{*}$ & 3.13 \\
\hline Harvest-index (\%) & 1.25 & $3.83^{* *}$ & 1.07 \\
\hline Grain yield / plant (g) & 1.25 & $1.63^{*}$ & 0.50 \\
\hline
\end{tabular}

Significance Levels $*=<.05, * *=<.01 \& * *$ 
Table.2 Heterobeltiosis (HB) and Standard heterosis (SH) for yield attributing traits in barley

\begin{tabular}{|c|c|c|c|c|c|c|c|c|c|c|c|}
\hline \multirow[t]{2}{*}{ S.N } & \multirow[t]{2}{*}{ Crosses } & \multicolumn{2}{|c|}{$\begin{array}{l}\text { Days to } 50 \% \\
\text { ear emergence }\end{array}$} & \multicolumn{2}{|c|}{$\begin{array}{l}\text { Days to } 75 \\
\text { \%maturity }\end{array}$} & \multicolumn{2}{|c|}{$\begin{array}{c}\text { Productive } \\
\text { Tillers/ Plant }\end{array}$} & \multicolumn{2}{|c|}{ Ear Length (cm) } & \multicolumn{2}{|c|}{ Grains/ Spike } \\
\hline & & HB & SH & HB & SH & HB & SH & HB & SH & HB & SH \\
\hline 1 & EC-667377×RD-2552 & -1.03 & -1.03 & $-3.20 *$ & -0.82 & $14.29 *$ & $14.29 *$ & 4.56 & 4.56 & $-12.32 *$ & $-10.87 *$ \\
\hline 2 & EC-667377×NDB-3 & $7.69 *$ & 1.03 & $-4.37 *$ & $-1.23 *$ & $24.19 *$ & $21.01 *$ & -7.61 & 1.40 & $-7.29 *$ & -5.77 \\
\hline 3 & EC-667377×Azad & $7.14^{*}$ & 0.52 & -1.94 & $3.69 *$ & $12.71 *$ & $13.45^{*}$ & -10.86 & -13.21 & $-24.96^{*}$ & $-23.72 *$ \\
\hline 4 & EC-667365×RD-2552 & -2.58 & -2.58 & 0.40 & 2.05 & -5.88 & -5.88 & -5.53 & 3.74 & $16.23 *$ & $-16.23 *$ \\
\hline 5 & EC-667365×NDB-3 & 0.00 & 0.00 & -0.40 & $2.87 *$ & -4.84 & -0.84 & 13.09 & -4.56 & -4.02 & -5.11 \\
\hline 6 & EC-667365×Azad & 0.00 & 0.00 & -1.55 & $4.10^{*}$ & -12.07 & $-14.29 *$ & $24.16 * *$ & -16.72 & $-14.32 *$ & $-15.98 *$ \\
\hline 7 & EC-667526×RD-2552 & -1.03 & -1.03 & -2.33 & $3.28 *$ & -7.56 & -7.56 & -0.86 & -0.82 & $21.05^{*}$ & $11.86^{*}$ \\
\hline 8 & EC-667526×NDB-3 & $4.37 *$ & -1.55 & $-5.04 *$ & 0.41 & -3.23 & 0.84 & -8.15 & -0.82 & $-19.28 *$ & $-9.88 *$ \\
\hline 9 & EC-667526×Azad & 2.19 & $-3.61 *$ & 0.00 & $5.74 *$ & 3.45 & 0.84 & 9.16 & 3.10 & $-18.87^{*}$ & $-9.43 *$ \\
\hline 10 & EC-667458×RD-2552 & -0.52 & -0.52 & -0.39 & $5.33 *$ & 7.56 & 7.56 & -10.51 & -5.55 & -3.08 & -3.08 \\
\hline 11 & EC-667458×NDB-3 & -0.52 & -1.03 & -1.55 & $4.10 *$ & -14.528 & -10.92 & $-17.89 *$ & -9.88 & -4.93 & $-6.01 *$ \\
\hline 12 & EC-667458×Azad & 3.11 & 2.58 & 0.00 & $5.74 *$ & -12.07 & $-14.29 *$ & -2.27 & 3.16 & $-18.02 *$ & $-19.60 *$ \\
\hline 13 & EC-667498×RD-2552 & $-4.57 *$ & -3.09 & 2.35 & $6.97 *$ & -7.14 & -7.14 & $16.54^{*}$ & -16.54 & $-21.21 *$ & $-21.21 *$ \\
\hline 14 & EC-667498×NDB-3 & -1.52 & 0.00 & 1.57 & $6.15^{*}$ & $22.58^{*}$ & $19.33^{*}$ & $14.32 *$ & -5.96 & 1.48 & 0.33 \\
\hline 15 & EC-667498×Azad & -1.02 & 0.52 & -0.39 & $5.33 *$ & 6.90 & 4.20 & 5.66 & -11.62 & -0.38 & -2.31 \\
\hline 16 & EC-667509×RD-2552 & -1.53 & -0.52 & -0.39 & $4.10^{*}$ & -3.95 & -3.95 & -9.06 & -9.06 & $8.54^{*}$ & $7.18 *$ \\
\hline 17 & EC-667509×NDB-3 & 0.00 & 1.03 & -0.39 & $4.10 *$ & -3.23 & 0.84 & $-21.30 * *$ & -13.62 & $-6.98 *$ & -5.60 \\
\hline 18 & EC-667509×Azad & $-3.57 *$ & -2.58 & -1.94 & $3.69 *$ & 5.17 & 2.52 & 11.92 & -3.39 & $-6.79 *$ & -5.41 \\
\hline 19 & EC-667454×RD-2552 & 1.55 & 1.55 & $6.15^{*}$ & $6.15^{*}$ & 10.92 & 10.92 & -4.96 & -2.63 & $-9.39 *$ & $-9.39 *$ \\
\hline 20 & EC-667454×NDB-3 & $7.69 *$ & 1.03 & -0.40 & $2.87 *$ & -4.84 & -0.84 & $-14.86^{*}$ & -6.55 & -1.27 & -2.39 \\
\hline 21 & EC-667454×Azad & 0.00 & $-6.19 *$ & 0.39 & $6.15^{*}$ & $-17.80 *$ & $-18.49 *$ & -9.30 & -7.07 & $-6.09 *$ & $-7.91 *$ \\
\hline
\end{tabular}


Table.3 Heterobeltiosis (HB) and Standard heterosis ( $\mathrm{SH}$ ) for yield attributing traits in barley

\begin{tabular}{|c|c|c|c|c|c|c|c|c|c|}
\hline \multirow[t]{2}{*}{$\begin{array}{l}\mathbf{S} . \\
\mathbf{N}\end{array}$} & \multirow[t]{2}{*}{ Crosses } & \multicolumn{2}{|c|}{$\begin{array}{c}\text { Biological Yield/ } \\
\text { Plant (g) }\end{array}$} & \multicolumn{2}{|c|}{$\begin{array}{c}\text { Grain Yield/ Plant } \\
\text { (g) }\end{array}$} & \multicolumn{2}{|c|}{$\begin{array}{c}\text { Harvest Index } \\
(\%)\end{array}$} & \multicolumn{2}{|c|}{$\begin{array}{l}1000 \text { Grain Weight } \\
\text { (g) }\end{array}$} \\
\hline & & HB & SH & HB & SH & HB & SH & HB & SH \\
\hline 1 & EC-667377×RD-2552 & $18.40 *$ & $-18.40^{*}$ & $-25.60 *$ & $-25.60 *$ & $-10.00 *$ & $-8.52 *$ & $-21.79 *$ & $-12.86^{*}$ \\
\hline 2 & EC-667377×NDB-3 & -5.75 & $-13.21 *$ & $18.28 *$ & $-23.35^{*}$ & $-6.80 *$ & -3.41 & $-16.67 *$ & $-7.14 *$ \\
\hline 3 & EC-667377×Azad & $-12.61 *$ & $-19.53^{*}$ & $-18.88 *$ & $-22.64 *$ & $-12.47 *$ & -4.29 & $-17.95^{*}$ & $-8.57 *$ \\
\hline 4 & EC-667365×RD-2552 & $20.68 *$ & $-20.68 *$ & $-18.58 *$ & $-18.58 *$ & 2.33 & 2.33 & $10.00 *$ & $10.00^{*}$ \\
\hline 5 & EC-667365×NDB-3 & 7.37 & -5.29 & 9.33 & 0.14 & 1.94 & 5.65 & 1.52 & -4.29 \\
\hline 6 & EC-667365×Azad & -1.01 & $-13.70 *$ & -9.41 & -13.61 & $-9.16^{*}$ & -0.68 & -4.29 & -4.29 \\
\hline 7 & EC-667526×RD-2552 & $16.33^{*}$ & $-16.33^{*}$ & $-17.19 *$ & $-17.19 *$ & -5.70 & -1.60 & $-7.14^{*}$ & $-7.14 *$ \\
\hline 8 & EC-667526×NDB-3 & -9.91 & $-17.54 *$ & -7.58 & -11.56 & 3.20 & $7.68 *$ & $13.64 *$ & $7.14^{*}$ \\
\hline 9 & EC-667526×Azad & -9.58 & $-17.24 *$ & $-18.06^{*}$ & $-21.59 *$ & $-11.08 *$ & -2.77 & $-12.86^{*}$ & $-12.86^{*}$ \\
\hline 10 & EC-667458×RD-2552 & -9.98 & -9.98 & $-14.42 *$ & $-14.42 *$ & -5.47 & -5.47 & -4.29 & -4.29 \\
\hline 11 & EC-667458×NDB-3 & $12.31 *$ & $-22.26^{*}$ & -14.70 & $-21.87 *$ & -3.51 & 0.00 & -3.08 & $-10.00 *$ \\
\hline 12 & EC-667458×Azad & 2.11 & -9.46 & -6.86 & -11.17 & $-9.11 *$ & -0.61 & $-12.86^{*}$ & $-12.86^{*}$ \\
\hline 13 & EC-667498×RD-2552 & $-21.86^{*}$ & $-21.86^{*}$ & $24.64 *$ & $-24.64 *$ & -4.53 & -4.53 & $-9.59 *$ & -5.71 \\
\hline 14 & EC-667498×NDB-3 & -9.68 & $-20.34 *$ & -15.02 & $22.16^{*}$ & $-7.71 *$ & -4.36 & -5.48 & -1.43 \\
\hline 15 & EC-667498×Azad & 4.44 & -8.95 & 4.26 & $-0.57 *$ & $-7.13 *$ & 1.54 & $-12.33^{*}$ & $-8.57 *$ \\
\hline 16 & EC-667509×RD-2552 & $20.44 *$ & $-20.44 *$ & $-23.88 *$ & $23.88^{*}$ & -5.98 & -5.98 & $-14.33^{*}$ & -4.54 \\
\hline 17 & EC-667509×NDB-3 & $-20.66^{*}$ & $-24.16^{*}$ & $-22.83 *$ & $-28.32 *$ & 0.41 & 4.06 & $-7.69 *$ & 2.86 \\
\hline 18 & EC-667509×Azad & $-14.49 *$ & $-18.26^{*}$ & $-19.08 *$ & $-22.83 *$ & $-9.25^{*}$ & -0.77 & 0.00 & $11.43^{*}$ \\
\hline 19 & EC-667454×RD-2552 & $-12.65^{*}$ & $-12.65^{*}$ & $-15.00 *$ & $-15.00 *$ & -0.57 & -0.57 & $-10.00^{*}$ & $-10.00 *$ \\
\hline 20 & EC-667454×NDB-3 & 5.33 & -7.09 & -5.21 & -13.18 & $-10.71 *$ & $-7.47 *$ & $-13.04 *$ & $-14.29 *$ \\
\hline 21 & EC-667454×Azad & $15.12 *$ & $-26.01 *$ & $-24.44 *$ & $-27.94 *$ & $-12.74 *$ & -4.59 & $-11.43^{*}$ & $-11.43 *$ \\
\hline
\end{tabular}

Significance Levels $*=<.05, * *=<.01 \& * *$ 
Increase in yield is the prime objective of any breeding program. The yield per plant trait showed desirable significant positive heterobeltiosis in two cross combinations viz., EC-667377 $\times$ NDB-3 and EC-667498 $\times$ RD2552. While significant standard heterosis was found in cross combinations: EC$667377 \times$ NDB-3, EC-667498 $\times$ NDB-3, and EC-667509×RD-2552 (Table-3). Higher magnitude of heterotic response for seed yield in barley has been reported earlier in barley by Singh et al., (2002), Rugen et al., (2004), Huang et al., (2007), Vishwakarma et al., (2011), Saad et al., (2013), Shendy (2015), Mansour (2016), and Ram and Shekhawat (2017).

In conclusion the present findings, the number of cross combinations with significant heterosis in desirable direction was few which can be attributed to parental materials used in the experiment. Nevertheless, the cross combinations which showed heterosis for yield and yield attributing traits in higher magnitude and in desirable direction can be utilized in future barley breeding program.

\section{References}

Amer, Kh. A., Eid, A. A., El-Akhdar, A. A. and El-Shawy, E.E. (2012). Combining ability and heterosis in five barley genotypes for some economic traits. Egy. J. Agric. Res., V. 90 (4): 105-116.

Eid, A. A. (2006). Breeding studies on some barley diseases. Ph.D. Thesis, Fac. Agric. Menofiya. Univ. Egypt.

El-Mouhamady, A.A., El-Seidy, E.H. and Aboud, K.A. (2012). Identification of a molecular markers linked to drought tolerance in some genotypes of barley. Aust. J. Basic \& Appl. Sci., 6(5):196-205.

Fonseca, S., Patterson F. L. (1968). Hybrid vigour in seven parent diallel crosses in common winter wheat (Triticum aestivum L.). Crop Sci. 8: 85-95.

Huang, Z., Li, Z., Rugen, X., Chao, L. (2007). Heterosis and combining ability for glume opening in Barley. J. Tritic. Crops. 27(1): 30-34.

Lal, C., Shekhawat, A.S., Singh, J. and Kumar, V. (2018).Study of heterosis in six-rowed barley (Hordeum vulgare L.). J. Pharmac. Phytochem., 7(5): 2287-2292.

Mansour, M. (2016). Genetical analysis of some quantitative traits in barley under saline soil conditions. Proceedings, The sixth field crops conference, FCRI, ARC, Giza, Egypt. 99: 107.

Panse, V.G., and Shukatme, P.V. 1967. Statistical methods for agricultural Workers, ICAR, New Delhi, India.

Parashar, N., Gothwal, D.K., Singh, G., Bhakal, M., Kumar, R. and Sharma, V., Heterosis Studies in Barley (Hordeum vulgare L.) Under Heat Stress Environment, Int. J. Pure App. Biosci. 7 (1): 183-189 (2019)

Ram, M., Shekhawat, A.S. (2017). Genotypic variances and interactions with environments in barley genotypes for grain yield and its associated characters. The Bioscan. 11(4):31733175.

Rugen, X.U. Chao, L.U., Huidmg, Z.M. (2004). Studies on the heterosis of barley (Hordeum vulgare L.). J. Act. Agronom. Sinic., 30: 668-674.

Saad, F. F., Abd El-Mohsen, A. A., Abd ElShafi, M. A. and Al-Soudan, I. H. (2013). Genetic behavior of grain yield and its components in barley crosses under water stress and non stress conditions. Sci. Agri. 1(2), 4555.

Shendy, M.Z. (2015). Estimation of heterosis, inbreeding depression and combining ability in six barley genotypes in both 
$\mathrm{F}_{1}$ and $\mathrm{F}_{2}$ generations. Eg. J. Pl. Breed. 19(4):167-182.

Singh, S.C. Singh, S.K. and Singh, S.K. (2002). Combining ability and heterosis in six-rowed barley. Progr.

Agric., 2: 54-56.
Vishwakarma, S.R., Shukla, A., Bahadur, R., Singh, N. (2011). Expression of heterosis for yield and chlorophyll content in barley. Pl. Arch., 11(2): 894.

\section{How to cite this article:}

Ankush Katiyar, Aarti Sharma, Akanksha Srivastava, Snehanshu Singh and Vishwakarma, S. R. 2020. Heterosis for Yield and Yield Attributing Traits in Barley (Hordeum vulgare L.). Int.J.Curr.Microbiol.App.Sci. 9(10): 248-254. doi: https://doi.org/10.20546/ijcmas.2020.910.032 